\title{
X-RAY NOVAE AND RELATED SYSTEMS
}

\author{
J. CRAIG WHEELER, SOON-WOOK KIM \\ Department of Astronomy \\ University of Texas \\ Austin, Texas 78712 \\ U.S.A.
}

and

SHIN MINESHIGE
Department of Physics
Ibaraki University
Mito, Ibaraki 310
JAPAN

\begin{abstract}
Accretion disk thermal instability models have been successful in accounting for the basic observations of dwarf novae and the steady behavior of nova-like systems. Models for the dwarf-nova like variability of the old nova and intermediate polar GK Per give good agreement with the burst amplitude, profile and recurrence time in the optical and UV. A monthlong "precursor plateau" in the UV is predicted for the expected 1992 outburst prior to the rise to maximum in the optical and UV. The models for the time scales of the outbursts and corresponding UV spectra at maximum are consistent with the inner edge of the accretion disk being essentially constant between quiescence and outburst and a factor of four larger than the corotation radius. These conclusions represent a challenge to the standard theory of magnetic accretion. Disk instability models have also given a good representation of the soft X-ray and optical outbursts of the X-ray novae A0620-00 and GS2000+25. Formation of coronae above the disk, heated by magneto-acoustic flux from the disk, may account for the temporal and spectral properties of the hard X-ray and gamma ray emission of related sources such as Cyg X1, GS 2023+33 (V404 Cyg), 1E 1740.7-2942 (the "Galactic Center" Einstein Source), and GS 1124-683 (Nova Muscae).
\end{abstract}

\section{Introduction}

A variety of accreting systems contain accretion disks around different types of compact objects. Some of these accretion disks are intrinsically unstable and a study of their behavior will help to better understand both the behavior of the accretion disks and the nature of the compact object.

Among the systems of interest are the novae and nova-like variables. These have a white dwarf and steady state disks. The dwarf novae also have white dwarfs but the characteristic 
variability of these systems is thought to be due to an intrinsic thermal instability in the accretion disk (Papaloizou, Faulkner and Lin 1983; Mineshige and Osaki 1983, 1985; Cannizzo and Wheeler 1984; Smak 1984; Meyer and Meyer-Hofmeister 1984). The intermediate polars are a subclass of the first two in which the white dwarf is magnetized. Among these GK Per, at least, also has an unstable accretion disk, as will be argued below. Certain classes of transient X-ray sources are also interesting in this regard. Soft X-ray transients like Aql X-1 and Cen X-4 are known to have Type I X-ray bursts and hence to be neutron stars, but they also display a longer term variability that is likely to be related to the dwarf nova accretion disk instability. A0620-00 is another soft X-ray transient that displays outbursts on time scales of decades that may be related to the dwarf nova thermal instability, but the compact object is thought to be a black hole (McClintock and Remillard 1986). Cygnus X-1, the classic black hole candidate, differs from A0620-00 because it has a high mass companion and a strong persistent $\mathrm{X}$-ray flux due to the high rate of mass transfer, but Cyg X-1 also shows occasional soft X-ray outbursts that are reminiscent of those in A0620-00. Therefore Cyg X-1 is also a candidate for hosting an unstable accretion disk. Finally, there is the long-standing suspicion that active galactic nuclei involve accretion into a supermassive black hole. On a larger scale, these systems, too, may be susceptible to a disk thermal instability (cf. Mineshige and Shields 1990). A principle long range goal of this research is to develop diagnostics that will allow one to differentiate neutron star from black hole binaries and to definitively test the hypothesis that AGN harbor black holes.

The theory of accretion disk limit cycle thermal instability is based on the physics of the vertical disk structure. In some regimes, the temperature rises as the surface density of the disk is increased in thermal equilibrium. For disk midplane temperatures corresponding to the ionization of hydrogen and helium, however, the surface density must decline as the bound-free opacity reaches a maximum with increasing temperature in order to maintain thermal equilibrium. The result is an "S-shaped" curve in the temperature/surface density plane. The portion of the thermal equilibrium curve with negative slope can be shown to be thermally unstable and that instability can trigger global heating and cooling waves (Lin, Papaloizou, and Faulkner 1985; Cannizzo, Wheeler, and Polidan 1986; Mineshige 1988). The instability depends somewhat on the assumed, and unknown, viscosity in the disk, but its basic existence is quite general since it depends primarily on the atomic physics of hydrogen. The resulting disk dynamics have given a reasonable account of dwarf nova instabilities, although there are still some interesting problems, for instance regarding the variation of the observed recurrence times (Cannizzo, Shafter, and Wheeler 1987).

An important feature of this class of models is that they are intrinsically time dependent. For steady state accretion disks, the flux from the disk surface is not a function of the viscosity parameter, $\alpha$, which is a dimensionless parameter relating the viscous stress tensor to the local pressure, $t_{\phi r}=\alpha P$ (Shakura and Sunyaev 1973). For a time-dependent situation, however, the flux does depend on the viscous dissipation. This is both an advantage and a disadvantage. The disk instability models do then become dependent on the recipe for the $\alpha$ parameter, but this also means that by studying time-dependent disks one can learn about the behavior and hence the physical nature of the viscosity. Study of dwarf novae shows that a single value of $\alpha$ does not fit the observations, so that $\alpha$ must be a function of conditions in the disk and variable with radius. A prescription that is empirically successful is $\alpha \propto(\mathrm{h} / \mathrm{r})^{\mathrm{n}}$, where $\mathrm{n} \geqslant 1$ (Mineshige and Wheeler 1989). This form and scale is also indicated by recent work on the fundamental fluid dynamics of the angular momentum transport process (Vishniac, Jin, and Diamond, 1990). These studies show that the angular momentum transport process is non-local, as indicated by the presence of the global parameter, $r$, in the prescription. In any case, a critical lesson of the study of such 
unstable accretion disks is that the mass flow rate through the inner edge of the disk, which determines the hard flux arising from the compact object, is not necessarily equal to the mass flow rate through the disk at an arbitrary intermediate radius, and neither mass flow rate is necessarily equal to the transfer rate from the companion star. In the case of an unstable disk, the latter is essentially never true. The flow rate in the disk is much higher than the transfer rate in outburst and much lower than the transfer rate in quiescence.

Although the basic physics is similar and a common parametrization of the viscosity is adopted, the disk instability models can display a rather large range of behavior. There are a number of variables in the problem that affect the outcome. The mass transfer rate into the disk is a key one. The recurrence time is roughly inversely proportional to the input rate, but the situation is more complicated than that because for decreasing input rates there is also a transition from having outbursts triggered in the outer parts of the disk to outbursts triggered in the inner parts of the disk. The latter occur when the viscous time scale in the disk is shorter than the input time scale so matter has a chance to flow appreciably during quiescence. The outer radius of the disk is significant because it sets the scale of the problem. Larger disks are more prone to insideout heating bursts. In general, neutron star and black hole systems tend to have larger orbital periods and hence larger disks than white dwarf systems. The inner radius of the disk also varies. It is larger for white dwarfs than for neutron star or black hole systems, and larger in magnetized than non-magnetized systems. A larger inner radius also tends to promote larger recurrence times, but the effect depends on the input rate and whether one is dealing with insideout or outside-in bursts. The mass of the central object also plays a role, with a larger mass tending to promote larger recurrence times, all else being the same. As discussed below, one also expects the behavior of various systems to diverge even more as the feedback of irradiation on the disk is incorporated. For basic, unirradiated models, one still gets an interesting range of behavior. For dwarf nova models with transfer rates of order $10^{-10} \mathrm{M} \odot / \mathrm{yr}$, inner and outer radii of $\sim 10^{9}$ and $\sim 3 \times 10^{10} \mathrm{~cm}$, respectively, and a $1 \mathrm{M}_{\odot}$ central object, the models typically give only outside-in bursts and recurrence times of a few months, as observed. For a system like A0620-00, one has transfer rates of order $10^{-11} \mathrm{M} \odot / y r$, inner and outer radii of $\sim 10^{6}$ and $\sim 10^{11}$ $\mathrm{cm}$, respectively, and a $10 \mathrm{M}_{\odot}$ central object, and the models give bursts that erupt within the disk (typically $\sim 10^{10} \mathrm{~cm}$ ) and propagate both directions with recurrence times of years to decades. The main difference is the lower mass input rate, but the larger outer and smaller inner disk radii and the larger central mass all contribute to giving the larger recurrence times for the same viscosity prescription.

\section{GK Persei}

GK Per displays a broad variety of cataclysmic variable activity. It was a classical nova in 1901, it has displayed semi-regular dwarf nova-like outbursts for decades and it is a magnetic accretor, showing a 351 second rotation period in the $\mathrm{X}$-ray flux. The rotation of the white dwarf is faster than the orbital period, defining GK Per as an intermediate polar. The companion is a sub-giant of about $1 / 2 \mathrm{M}_{\odot}$ and the orbital period is very close to 2 days. This large orbit gives a large outer radius for the disk. We take the mass of the white dwarf to be $\sim 0.7 \mathrm{M} \odot$.

The dwarf nova-like outbursts show a rise from about magnitude 13 to magnitude 10 with a recurrence time of 800 - 1200 days. Kim, Mineshige, and Wheeler (1992) have modeled these outbursts with an accretion disk instability. For a mass transfer rate of about $10^{-8} \mathrm{M} / \mathrm{yr}$ and an inner disk radius of about $3 \times 10^{10} \mathrm{~cm}$ the flares are well reproduced. 
The result for the inner radius is especially interesting. With the standard magnetosphere boundary condition, in which, heuristically, the ram pressure of the Keplerian flow of the disk balances the magnetic pressure in the magnetosphere (Pringle and Rees 1972; Davidson and Ostriker 1973; Lamb, Pethick, and Pines 1972), the magnetic field of the white dwarf would have to be about $10^{7}$ Gauss, comparable to that in AM Her stars (polars). The current result for the inner boundary is not, however, consistent with this theory of the inner boundary. The inner boundary of the disk derived from the instability calculations is about 4 times larger than the corotation radius, the position of zero torque between the disk and the magnetosphere where the Keplerian frequency in the disk matches the rotation frequency of the white dwarf. According to this standard theory, accretion should be prevented by an angular momentum barrier if the disk boundary exceeds the co-rotation radius, and yet accretion occurs both in outburst and quiescence as shown by the X-ray flux. The basic magnetic boundary theory also predicts that the inner radius of the disk should be modulated by the mass flow through the disk, $r \propto \dot{M}^{-2 / 7}$. The results of these models suggest, however, that the inner radius of the disk moves very little even though the mass flow through the inner edge of the disk fluctuates by a factor of $\lesssim 1000$ from quiescence to outburst, as will be argued below.

The instability models show that the disk is expected to be rather cool and dim in quiescence, much dimmer than the 13th magnitude observed for the system. This luminosity is presumably provided by the companion and hot spot. The models also show a behavior that is peculiar to the disk of GK Per with its large inner and outer radii. The disk shows repeated phases when it is heated to a warm intermediate, thermally unstable state, but does not heat all the way to the highest, hottest state. Such a precursor burst should trigger an increased mass flow onto the magnetosphere and white dwarf and an increase in the UV and X-ray luminosity, but it is predicted to be too dim to be seen in the visual against the 13th magnitude background. Finally, one of these intermediate temperature flares is followed immediately by a full heating wave and a rise to the bright, visually observable maximum. The latter behavior is always accompanied by a long precursor plateau in the mass flow rate onto the white dwarf and then a brief spike before the final rise to maximum. The precursor plateau is illustrated in Figure 1. This phase can last for a

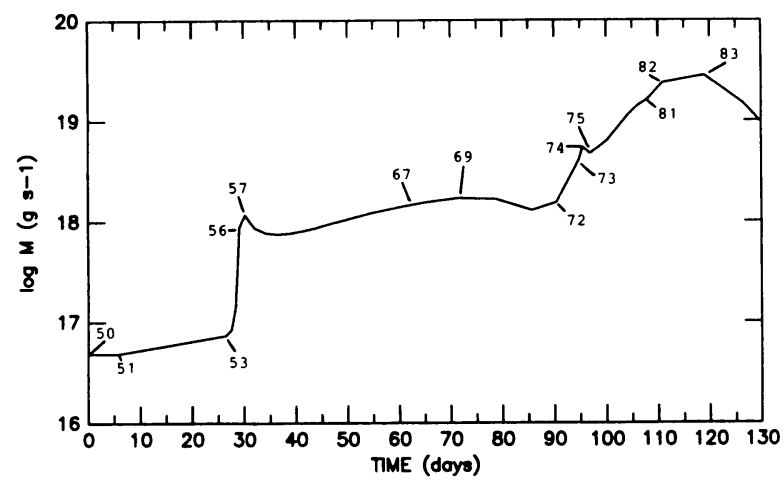

Figure 1. The calculated mass flow rate through the inner edge of the disk is shown for a typical model of GK Per. The numbers represent time steps in the model. 
month or more and should show up in the UV flux before any sign of the optical outburst. The brief flare and pause on the rise to maximum should also be revealed in the UV flux and perhaps in the optical. The lower left panel of Figure 2 shows the predicted profile in the mass flow rate and the upper panel shows the UV flux from the 1981 outburst that appears to reflect the computed behavior. The lower middle panel of Figure 2 shows the model optical outburst which does not display the precursor halt in this particular model, but the observed optical bursts of the other three panels show that such a feature may appear in the observations.

In these calculations the inner edge of the disk is formally held constant (for reasons of numerical stability), but the value of the inner radius of the disk where the density is formally required to go to zero is varied as a parameter to fit, among other things, the recurrence time.
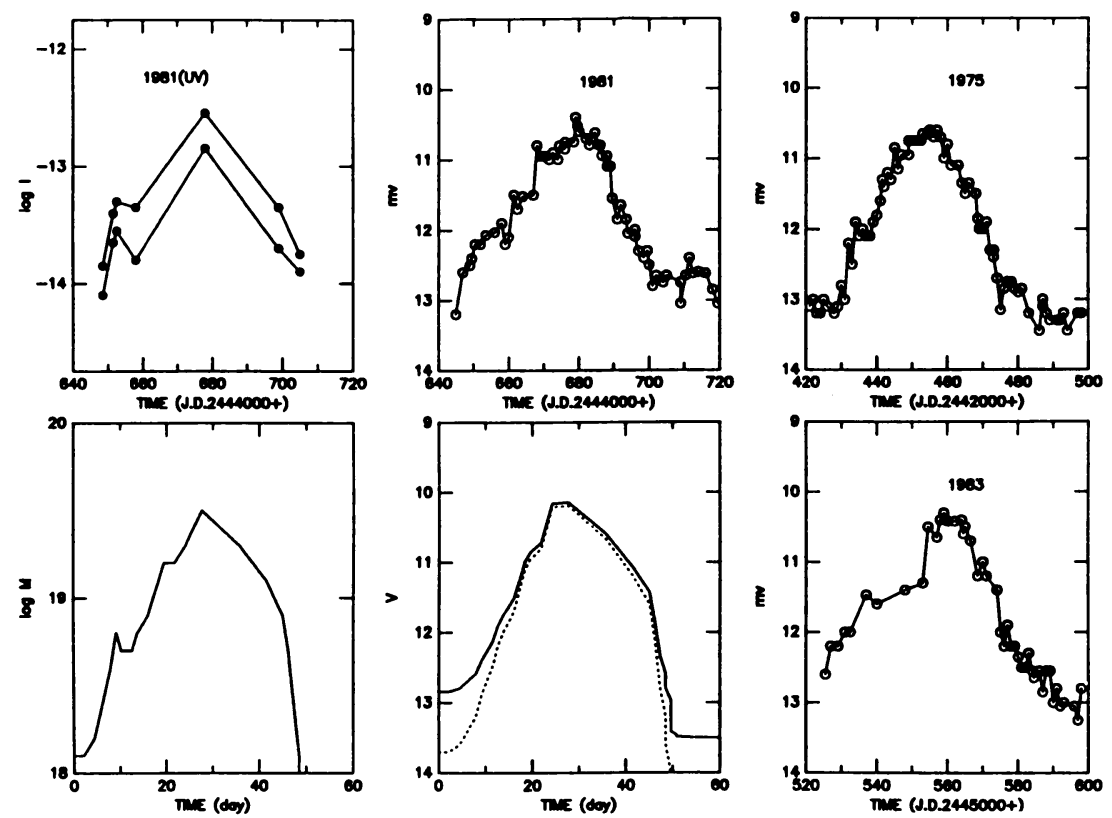

Figure 2. The two upper left panels show the UV (1640 $\AA$ and $1240 \AA$ ) and optical observations of the 1981 outburst (Bianchini et al. 1986) and the right hand upper and lower panels light curves from 1975 and 1983, respectively (Szkody, Mattei, and Mateo 1985). The other two lower panels give the inner mass accretion rate and optical flux for the model of Figure 1 . In the model optical light curve, the dashed line represents the flux from the disk and the solid line the addition of a constant source of $13 \mathrm{~m}^{\mathrm{m}}$. 
Since the recurrence time is a feature of the quiescent disk it seems most appropriate to identify the inner radius of the models with that in quiescence. We can independently argue, however, that the radius does not vary appreciably in outburst and that the radius is almost the same as in quiescence. The lower panel of Figure 3 shows the evolution of the outburst continuum spectrum, obtained by fitting Kurucz (1979) atmospheres to the disk, from the onset of the outburst to maximum. The upper panel shows the observed UV/optical continuum near maximum. Note that the observed flux is rather flat, as is the predicted spectrum near maximum. The latter is a rather sensitive function of the inner radius of the disk. Similar models in which the inner radius of the disk is assumed to extend to within the co-rotation radius show a steep rise in the UV in violation of the observations (Mauche et al. 1990). While a slightly smaller inner radius might be compatible with the observed spectrum, a reduction of the inner radius by a factor of 4 to bring it within the co-rotation radius does not seem to be allowed. If there is disk material between $\sim 3 \times 10^{10} \mathrm{~cm}$ and the co-rotation radius, then it does not seem to participate in the disk instability and it does not radiate in the UV during the outburst.
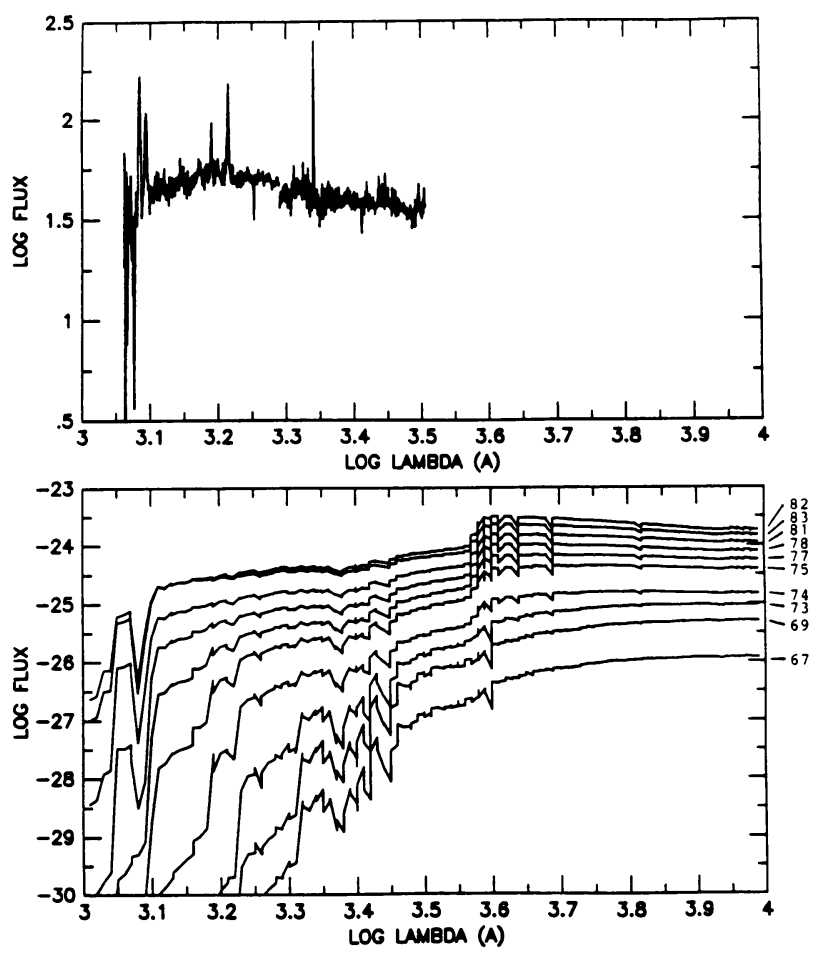

Figure 3. Time-dependent spectra based on Kurucz atmospheres for the model of Figures 1 and 2. The numbers represent the stages labeled in Figure 1. The upper spectrum corresponds to the observations of GK Per near maximum in the 1981 outburst from Wu et al. (1989). 
Another interesting feature of the observations of GK Per is that the observed $2-10 \mathrm{keV} \mathrm{X}$ ray flux does not change by more than a factor of ten from quiescence to outburst. As noted above, the disk models predict a fluctuation of the mass flow rate through the inner edge of the disk by a factor of $\lesssim 1000$. Yi, Kim, Vishniac, and Wheeler (1992) resolve this paradox by noting that the system is nearly edge on. In the outburst models, the full luminosity of the disk is very bright and the peak magnitude of 10 is achieved by adopting a sufficient inclination. The result is $\mathrm{i} \sim 84^{\circ}$. This is large, but not incommensurate with other estimates of the inclination. Under these circumstances, Yi et al. argue that the accretion column or fan stands up above the white dwarf a distance comparable to the radius of the white dwarf so that the entire quiescent $\mathrm{X}$ ray flux can be seen above the relatively thin disk. In outburst, the disk is thicker and the accretion column is compressed down onto the white dwarf. The result is that the X-rays are blocked and can not be observed directly. Only X-rays that propagate up the accretion column and are scattered into the line of sight can be observed in outburst. Yi et al. estimate that about one percent of the total flux could be scattered, consistent with an observed increase of about a factor of 10 over quiescence.

Thus the disk instability model gives a good representation of the dwarf nova-like outbursts of GK Per in terms of the amplitude, duration, recurrence time, details of the peak profiles in the UV and optical and the behavior of the X-ray flux. The models predict UV and perhaps X-ray activity up to two months prior to the optical outburst that should occur in 1992. Preparations should be made to observe this predicted precursor hard flux.

The instability models and the UV spectrum show that the radius of the inner edge of the disk is about 4 times the co-rotation and does not vary appreciably from outburst to quiescence. This calls into question the standard magnetic accretion theory which predicts that no accretion onto the white dwarf should occur in these circumstances and that the inner edge of the disk should vary appreciably. This is of potentially great significance because if this boundary theory is incorrect for GK Per it may be as well for accreting magnetic neutron stars where it has received broad application.

\section{X-ray Transients}

The success of the application of the disk instability model to GK Per gives more confidence in applying the theory to more exotic and demanding circumstances. These models have been used to explore the black hole candidate A0620-00 (Huang and Wheeler 1989; Mineshige and Wheeler 1989). The result was that for appropriate parameters, the heating instability occurred naturally to interrupt the quiescence in the disk. The burst magnitude and duration in both the optical and the soft X-rays (assumed to scale with the mass flow rate through the inner edge of the disk) and the recurrence interval were similar to those observed for the system. The interval of decades between outbursts was consistent with a $10 \mathrm{M}_{\odot}$ central object, but not with one of $1 \mathrm{M}_{\odot}$. This provides some independent evidence that A0620-00 contains a black hole. The outer disk is predicted to be cold and dim with a surface temperature of about $3000 \mathrm{~K}$ in quiescence. This is consistent with the presence of an optically thick disk observed at some epochs (Haswell and Shafter 1990). The origin of the blue light in quiescence with a luminosity about half that of the subgiant companion is still not completely explained. It may come from hotter inner parts of the disk for some prescriptions of the viscosity parameter (Mineshige and Wheeler 1989) or there may be some contribution from the hot spot. There may be some form of chromosphere on the 
disk, but the observation of emission lines (Johnston, Kulkarni, and Oke 1989) does not necessarily mean that the whole disk body is optically thin.

The disk models give about the right amplitude for the optical outburst for A0620-00 and even the right slope for the decline from peak, but then the models decay too rapidly after about 100 days. The observed light curve may be extended by the effects of X-ray irradiation from the inner disk. Irradiation could also cause a more thorough dumping of the mass from the disk and hence lengthen the recharge and thus the recurrence time.

A number of other soft $\mathrm{X}$-ray transients have recently been discovered that have some properties in common with A0620-00 and hence are suspected of being black hole sources. The X-ray light curves of GS 2000+25, and GS $11124-68$ (Nova Muscae) were very similar Tanaka (1990 and this volume). The transient source GS 2023+338 (V404 Cyg) also had some similarities, but showed much larger fluctuations in its X-ray light curve. Mineshige, Kim, and Wheeler (1990) argue that the soft X-ray flux from the hot inner surfaces of their disk models reproduces the spectral behavior observed for GS $2000+25$, namely a cooling after outburst at nearly constant inner radius. This source also displayed a hard power-law component that developed after the maximum in the soft X-ray light curve and fluctuated independently of the soft $\mathrm{X}$-ray flux intensity. A0620-00 showed roughly the same behavior, with a hard component that developed after the soft- X-ray peak. V404 Cyg did not show the soft component, but only the hard power law that decayed fairly monotonically. V404 Cyg showed evidence for large X-ray absorption so it is possible that the system is nearly edge-on and the soft X-ray flux from the disk surface can not been seen directly. Tanaka argues, however, that the variation in the intensity of the hard power-law component is not caused entirely by variable absorption, but is intrinsic to the system. In other black hole candidates the flickering of the hard component is anticorrelated with the soft X-ray flux. Tanaka concludes that the fluctuations in the hard component imply that the soft flux is intrinsically low, and not just obscured.

Another system of great interest in this regard is Cyg X-1. Cyg X-1 shows a variety of temporal behavior, but the high soft X-ray states are of particular interest in this regard. There was a high state in 1972, another in 1975 and a third in 1980 (Ling et al. 1987). None has been observed since. Cyg X-1 shows a hard power -law component that is very similar to those of the soft X-ray transients just mentioned. The high soft X-ray states are anticorrelated with the intensity of the hard component. The high states last for a few months and are very reminiscent of the outbursts of the soft X-ray transients. It is thus reasonable to inquire whether Cyg X-1 is also experiencing accretion disk instabilities and plausible models can be constructed (Wheeler $e t$ al. 1990). While the statistics are too poor for a firm conclusion, the spacing of the bursts, $\sim 3$ years, $\sim 5$ years, and $\gtrsim 10$ years, raises the question of whether there is some secular variation in the mass transfer rate toward smaller values.

Besides its overall similarity to A0620-00 and GS 2000+25, Nova Muscae is of particular interest because it has been observed to be a source of annihilation radiation (Sunyaev et al.1991; Sunyaev, Jourdain, and Goldwurm 1991). Such emission has long been associated with black holes, but it is not clear that it is uniquely so. In any case, the observations of Novae Muscae raise the interesting question of the origin of this radiation in a corona or other structure and whether such physical processes proceed in the other soft X-ray transients. The "Galactic center" Einstein source 1E 1740.7-2942 has recently been spatially resolved to be distinct from the Galactic center and confirmed as the long studied transient source of annihilation radiation toward the Galactic center (Sunyaev et al. 1991). There is a strong suspicion that this source harbors a black hole and it is of great interest to ask if its transient nature has any relation to an accretion 
disk instability. The implication is that an electron-positron dominated corona may be involved in all the black hole and perhaps other systems.

Further study of these systems requires the investigation of the irradiation of the disk by hard flux from the inner surface of the disk, the surface, if any, of the compact object, by flux reflected from or generated in any corona or otherwise extended structure, and perhaps flux from a hot companion star. This is, in general, a difficult, complexly coupled problem. Tuchman, Mineshige, and Wheeler (1990) and Mineshige, Tuchman, and Wheeler (1990) have addressed some of the basic issues involved. They showed that for soft X-rays that are absorbed in the outer layers of the disk, the disk is affected only in its outer layers when it is in the hot, radiative state. In quiescence, however, when the disk is expected to be convective, irradiation flux can affect the whole vertical structure of the disk. Tuchman et al. define an irradiation temperature according to the irradiation flux as:

$$
F_{i r r}=\frac{C L_{X}}{2 \pi r^{2}}=\sigma T_{i r r}^{4}
$$

where $\mathrm{C}$ is a geometric factor describing how much of the total luminosity from the central object falls on the disk surface in a given annulus. This factor might be $\sim 10^{-2}$ for direct radiation from the central object or $\sim 10^{-5}$ for indirect radiation reflected from a corona. Tuchman et al. conclude that for a system like A0620-00 the irradiation is negligible in quiescence, but is likely to be appreciable in outburst. For a source like Cyg X-1 irradiation is probably always a major factor because of the high mass transfer rate and hot disk and the bright, hot companion.

\section{Conclusions}

These studies have shown that the accretion disk thermal instability is a fundamental, ubiquitous phenomenon. It occurs in dwarf novae, in X-ray transients, in intermediate polars and probably in quasars. Study of the properties of the instability constrain the very presence of an accretion disk, the nature of the viscosity, the mass and nature of the central object, and the strength of the magnetic field of the central object and its interaction with the disk.

A major unsolved problem is the construction of self-consistent, time-dependent, irradiated accretions disks and associated coronae. The current accretion disk models give a new framework in which to study the latter. Rather than considering the corona as a separate component, the stage is now set to use the basic time-dependent disk structure that comes from the disk models to inquire about how this structure may interact with any corona. For instance, while coronae have been hypothesized to contain internal sources of viscous heating, it is very likely that they are powered by some portion of the vertical flux from the disk, for instance in the form of magnetoacoustic waves. Likewise, irradiation from such a corona can affect the disk structure. We are now in a position to study such problems and construct much more realistic models of the transient $\mathrm{X}$-ray sources and perhaps to gain qualitative new understanding of the nature of the annihilation radiation sources.

This research was supported in part by NSF Grant 8915754 and NASA Grant NAGW 1807. Numerical calculations were performed on the Cray Y-MP at the University of Texas System Center for High Performance Computing. 


\section{References}

Binachini, A., Hamsaoglu, E., and Sabbadin, F. 1981, Astr. Ap., 99, 392.

Cannizzo, J. K. and Wheeler, J. C. 1984, Ap. J. Suppl., 55, 367.

Cannizzo, J. K., Shafter, A. W., and Wheeler, J. C. 1988, Ap. J., 333, 227.

Cannizzo, J. K., Wheeler, J. C., and Polidan, R. S. 1986, Ap. J., 301, 634.

Davidson, K. and Ostriker, J. P. 1973, Ap. J., 179, 585.

Haswell, C. A. and Shafter, A. W. 1990, Ap. J. Lett., 359, L47.

Huang, M. and Wheeler, J. C. 1989, Ap. J., 343, 229.

Johnston, H. M., Kulkarni, S. R., and Oke, J. B. Ap. J., 345, 492.

Kim, S.-W., Wheeler, J. C., and Mineshige, S. 1992, Ap. J., in press.

Kurucz, R. L. 1979, Ap. J. Suppl., 40, 1.

Lamb, F. K., Pethick, C. J., and Pines, D. 1973, Ap. J., 184, 271.

Lin, D.N.C., Papaloizou, J., and Faulkner, J. 1985, M.N.R.A.S., 212, 105.

Ling, J. C., Mahoney, W. A., Wheaton, W. A., and Jacobsen, A. S. 1987, Ap. J. Lett., 321, L117.

Mauche, C. W., Miller, G. S., Raymond, J. C., and Lamb, F. K. 1990, in Accretion Powered Compact Binaries (Cambridge: Cambridge University Press), p.195.

McClintock, J. E. and Remillard, R. A. 1986, Ap. J., 308, 110.

Meyer, F. and Meyer-Hofmeister, E. 1984, Astr. Ap., 132, 143.

Mineshige, S. 1988, Ap. J., 335, 881.

Mineshige, S., Kim, S.-W., and Wheeler, J. C. 1990, Ap. J. Lett., 358, L5.

Mineshige, S. and Osaki, Y. 1983, Publ. Astron. Soc. Japan, 35, 377.

Mineshige, S. and Osaki, Y. 1985, Publ. Astron. Soc. Japan, 37, 1.

Mineshige, S. and Shields, G. A. 1990, Ap. J., 351, 241.

Mineshige, S., Tuchman, Y., and Wheeler, J. C. 1990, Ap. J., 359, 164.

Mineshige, S. and Wheeler, J. C. 1989, Ap. J., 343, 241.

Papaloizou, J., Faulkner, J., and Lin, D. N. C. 1983, M.N.R.A.S., 205, 487.

Pringle, J. E. and Rees, M. J. 1972, Astron. Ap., 21, 1.

Shakura, N. I. and Sunyaev, R. A. 1973, Astr. Ap., 24, 337.

Smak, J. 1984, Acta Astron., 34, 161.

Sunyaev, R. A., et al. 1991, IAUC 5176.

Sunyaev, R. A., Jourdain, E., and Goldwurm, A. 1991, IAUC 5201.

Szkody, P., Mattei, M., and Mateo, M. 1985, Publ. Astron. Soc. Pac., 97, 264.

Tanaka, Y. 1990 in The 23rd ESLAB Symposium on Two Topics in X-ray Astronomy, ed. N. White (ESA SP-296), p. 2.

Tuchman, Y., Mineshige, S., and Wheeler, J. C. 1990, Ap. J., 359, 164.

Vishniac, E. T., Jin, L., and Diamond, P. H. 1990, Ap. J., 365, 648.

Wheeler, J. C., Mineshige, S., Huang, M., and Kim, S.-W. 1990, in Cataclysmic Binaries and Low Mass X-ray Binaries, ed. C. Mauche (Cambridge: Cambridge University Press), p. 315.

Wu, C-C., Panek, R. J., Holm, A. V., and Raymond, J. C. 1989, Ap. J., 339, 443.

Yi, I., Kim, S.-W., Vishniac, E. T., and Wheeler, J. C. 1992, in preparation. 